\title{
Effects of Two Music Therapy Methods on Agitation and Anxiety among Patients Weaning off Mechanical Ventilation: A Pilot Study
}

\author{
Park, Jong Yoen ${ }^{1)} \cdot$ Park, Soohyun ${ }^{2)}$
}

\author{
1) Nurse Manager, Ewha Womans University Medical Center, Seoul \\ 2) Assistant Professor, Department of Nursing, Eulji University, Seongnam, Korea
}

\begin{abstract}
Purpose: The feasibility and differential effects of two music therapy methods (interventions with preferred music vs. classical relaxation music) were done to examine the effects on agitation and anxiety in patients weaning off mechanical ventilation. Methods: This pilot study was conducted using a crossover design. Six patients listened to preferred music choices and classical relaxation music. Anxiety scores were measured using the Richmond Agitation Sedation Scale (RASS), State-Trait Anxiety Inventory (STAI), and visual analog scale (VAS). Results: Patients showed a significant decrease in agitation and anxiety after both the preferred and classical relaxation music interventions. The difference in the effects of preferred music and that of classical relaxation music was not significant. As for feasibility, patients exhibited a change in agitated behaviors after the music interventions by not trying to take off medical devices and quietly listening to the music, and by smiling and moving lips along with the lyrics while listening. Conclusion: Music interventions which centered on either patients' preferences or classical relaxation music to enhance relaxation, helped reduce agitation and anxiety during the mechanical ventilation weaning process.
\end{abstract}

Key Words: Music; Agitation; Anxiety; Ventilation

*This article was supported by Eulji University in 2016.

Received Mar 22, 2019 Revised May 10, 2019 Accepted May 14, 2019

Corresponding author: Park, Soohyun Department of Nursing, Eulji University

553 Sanseong-daero, Sujeong-gu, Seongnam 13135, Korea

Tel: +82-31-740-7184, Fax: +82-31-740-7359, E-mail: soohyunp@eulji.ac.kr 


\section{INTRODUCTION}

Mechanical ventilation (MV) is typically used to treat acute respiratory failure. More than $20 \%$ of patients in intensive care units (ICUs) undergo MV [1]. Weaning off MV refers to the transition period of progressively reducing ventilator support [2]. During the weaning process, patients can experience severe anxiety, stress, fear, agitation, and lack of sleep [3-5]. These distressing experiences can increase the breathing workload and fatigue by stimulating the sympathetic nervous system, which can lengthen the weaning period $[4,6]$. Longer weaning periods can lead to longer hospitalizations and increased hospital costs [7]. To reduce costs and stays, healthcare providers should help patients successfully wean off MV in order to better recover spontaneous breathing after extubation [8].

In general, clinicians use pharmacological treatments to manage distressing experiences during MV weaning, such as analgesics and sedatives [7]. However, during the weaning period, the clinician also strives to keep patients alert by decreasing medication dosages of sedative medications in order to stimulate patients' spontaneous breathing. Since certain analgesics have side effects that include respiratory depression and weakening of the respiratory muscles, clinicians have been searching for effective non-pharmacological interventions [4]. Nonpharmacological approaches such as cognitive behavior therapy and respiratory muscle training have been used; however, these techniques require a trained professional $[8,9]$. Therefore, music therapy is used because it is inexpensive compared to sedative and analgesic medication and can be applied without any specialized training [10]. A study showed that ventilated patients are more likely to experience a successful weaning process when awake and aware of their surroundings [11]. Because music can increase patients' awareness of their surroundings through physiological and psychological stimulation, music therapy has been proposed as an environmental intervention [10].

Music therapy is a non-pharmacological, environmental intervention that has been effective for decreasing anxiety, stress, and agitation as well as increasing relaxation $[12,13]$. Specifically, music interventions have been shown to be effective for lowering ventilated patients' stress, anxiety, and agitation $[14,15]$. This is also true during the MV weaning process-many patients report decreases in stress and anxiety after music interventions [16-18]. However, thus far, no prior research has been conducted on the effect of music interventions on agitation for patients while weaning off MV. There is some evidence that sound-based therapies can help agitation. For instance, in a study using nature-based sound therapy-such as birdsongs, soothing rain sounds, river or waterfall sounds, or sounds of walking through the forest-patients reported significantly decreased agitation [19].

Furthermore, a music intervention was effective for decreasing patients' agitation during the MV period [15]. Thus, music interventions may help with managing agitation during the weaning process. In order to confirm this, we explored the feasibility of two types of music interventions (preferred and classical relaxation music) for improving patients' agitation in the present study.

The music utilized in most previous studies investigating the MV weaning process was selected by healthcare professionals and included classical music, relaxing music, or Korean traditional music $[16,17]$. While such music is intended to have a soothing effect, this might not be the case for all patients [14]. Therefore, it is necessary to explore whether music that better reflects patients' preferences is also suitable for music therapy. The findings from the previous study, which was the only study with patients' preferred music, indicated that preferred music was related to a decrease in anxiety and dyspnea compared to a no music condition [18]. Another notable gap in the literature is that no study has compared the intervention effects of patients' preferred music and healthcare providers' selected music (e.g., soothing music). Therefore, the present study also compared the effectiveness of preferred music and classical relaxation music in decreasing anxiety and agitation. In summary, the goals of the present pilot study were to identify the feasibility of preferred music and classical relaxation music interventions for reducing agitation and anxiety among patients who are weaning off MV and to compare any differences in the effectiveness of these two interventions.

\section{METHODS}

\section{Study Design}

This pilot study was conducted with a single group utilizing a crossover design. Patients were recruited from a surgical intensive care unit at a general hospital in Seoul, South Korea, from September 2017 to February 2018. Two different interventions-a preferred music intervention vs. a classical relaxation music intervention-were provided in a different sequence. Patients acted as their own controls in this study, as it is difficult to randomize individuals undergoing ventilation weaning into cross-matched groups. As there were likely to be some cumulative carryover effects from the first intervention to the second, patients 
were randomized into an intervention order-preferred music or classical relaxation music first-using a computer random number generator.

\section{Setting and Sample}

Patients' eligibility was identified by the ICU manager in the selected setting. To be eligible for this study, participants had to be (1) aged 19 years or older; (2) undergoing the pressure support ventilation (PSV) mode of MV; (3) alert; (4) capable of nonverbal communication; (5) hemodynamically stable during the weaning process, including having a partial pressure of oxygen ( $\mathrm{PaO} 2)$ / fraction of inspired oxygen $(\mathrm{FiO} 2)$ ratio over 200 , a heart rate of less than $140 \mathrm{bpm}$, and a mean blood pressure of more than $60 \mathrm{mmHg}$ [20]; and (6) able to listen to music via headphones without problems. Patients with a history of psychiatric illness, cognitive disorders, receiving continuous intravenous sedation, and neurological disorders were excluded. Applying the inclusion and exclusion criteria, six patients were included in the study.

\section{Measurements}

\section{1) Music Assessment Tool (MAT)}

The MAT, developed by Chlan and Heiderscheit [21], was used as a guide to select patients' preferred music in this study. This tool gathers comprehensive information about music preferences through five questions as follows: (1) whether a patient likes music or not; (2) whether a patient is professional music or not musician; (3) patients' preferences music types among 14 different genres of music; (4) specific artists or groups that a patient likes; and (5) genres of music that the patients do not like.

\section{2) Agitation}

Agitation was assessed using the Richmond Agitation Sedation Scale (RASS). The RASS is a single-item measure that uses a 10-point scale ranging from -5 to +4 . The RASS comprises four levels of anxiety or agitation (1-4), one level of a calm and alert state ( $(0)$, and five levels of sedation (-1 to -5$)$. Higher scores indicate a more agitated state. Validity of the RASS has been reported in previous studies, and the RASS is strongly correlated with the Sedation Agitation Scale score $(\mathrm{r}=.78, p<.001)$, Ramsay Sedation Scale $(\mathrm{r}=.78$, $p<.001)$, and Glasgow Coma Scale $(\mathrm{r}=.79, p<.001)$ [22].

\section{3) Anxiety}

Anxiety was assessed using the State-Trait Anxiety Inventory (STAI) [23]. The scale comprises 20 items rated on a 4-point Likert scale ranging from 1 ("strongly disagree") to 4 ("strongly agree"). The reverse-scored items are 1, 2, 5, $8,10,11,15,16,19$, and 20 . Total scores range from 20 to 80 , with higher scores indicating higher anxiety levels. The STAI shows good reliability-it had a Cronbach's $\alpha$ of 0.89 in a previous study [24] and 0.96 in the present study. Furthermore, the test-retest reliability coefficients for this scale range from .69 to .89 based on intervals ranging from 1 hour to 104 days [25].

Anxiety was also measured using a self-reported 10-mm visual analog scale (VAS). The VAS comprised a 10-cm horizontal line, the ends of which reflected the extremes of anxiety severity (the left end indicated no anxiety, and the right end indicated the highest anxiety).

\section{Interventions}

Preferred music was selected using the MAT, and questions were verbally delivered to patients by the researcher. Since the patients could not speak, the researcher had patients communicate their answers by writing on paper or using body language (e.g., head nods). Based on answers to the MAT, a list of each patient's favorite songs was created. The researcher then downloaded each of these songs from an online music streaming service.

For the classical relaxation music, an album called "Meditation: Classical Relaxation Vol. 3" was selected. This anthology includes a variety of composers, and most of the songs have a slow tempo and beat. The songs have been demonstrated to have a soothing effect [26].

When weaning trials first began, participants listened to their preferred music or the classical relaxation music for 30 minutes. Thirty minutes was selected in this study because 30 minutes has been employed in most studies on the effect of music therapy $[14,23,25]$. However, there is currently no consensus regarding the most effective duration. All patients listened to music via headphones connected to an MP3 player. The music volume was set to patients' preference. The researcher asked patients whether he/she wanted to change the volume, and then the patients answered this question by nodding (yes) or shaking (no) his/her head. For those responding "yes," the researcher changed the volume and asked if the changed volume was acceptable. All music interventions were performed between nursing procedures to prevent the interventions from being interrupted.

\section{Data Collection}

The researcher recorded the patients' general character- 
istics, such as age, education, and medical diagnosis, using medical records. Before music intervention, patients were administered the self-reported STAI and VAS anxiety scale. If a patient could not complete the STAI questionnaires, the researcher read the questions and patients answered by nodding their head when the researcher pointed to the corresponding number on the rating scale (1 to 4 ). For the VAS scale, patients pointed to a spot on the $10-\mathrm{cm}$ horizontal line with their finger. The RASS was completed by the researcher while observing the patient's status before the music intervention began. After completing each music intervention, the anxiety VAS scale, the STAI, and the RASS was completed according to the same methods that performed before the intervention.

The entire experiment lasted for 120 minutes. Three patients underwent the classical relaxation music intervention for the first 30 minutes, followed by the preferred music intervention for another 30 minutes. The other three patients received the preferred music intervention first, followed by the classical relaxation music, for the same length of time. There was a 60 minute washout period between the two music interventions. During the washout period, patients did not participate in any other research procedures (Figure 1).

During data collection, other sources of simulations, such as light in the room, were minimized (e.g., lights were dimmed), and healthcare providers were instructed not to interrupt patients during the music listening periods. Patients were lying quietly with their eyes closed during the interventions. The intervention was stopped if patients experienced any of the following: heart rate $<140 \mathrm{bpm}$ and mean blood pressure $>60 \mathrm{mmHg}$. No patients experi- enced these conditions during the intervention period.

\section{Data Analyses}

Statistical analyses were conducted using SPSS/WIN Statistics 21.0 for Windows. Demographic characteristics were analyzed using descriptive statistics. To compare medians of agitation and anxiety scores between the interventions, Mann-Whitney U tests and Wilcoxon signed rank tests were used.

\section{Ethical Considerations}

The study was approved by the Institutional Review Board (IRB: 2016-08-059) of the general hospital where we collected the data. The researcher explained the benefits and risks of this study to both the patients and family members. If patients and their family members agreed to participate, informed consent was obtained from both the patient and the family members. Patients were informed of their anonymity and confidentiality, and patients could withdraw from the study at any time.

\section{RESULTS}

\section{Demographic Information}

More than half of the patients were female, and mean age was 45.33 years (Table 1 ). The mean number of days of MV was 10.67. All patients were taking pain medication. Most participants preferred either trendy Korean pop music or Korean oldies music; classical music was not selec-

\section{Preferred music 30 minutes

Wash-out
60 minutes
Classical relaxation music
30 minutes
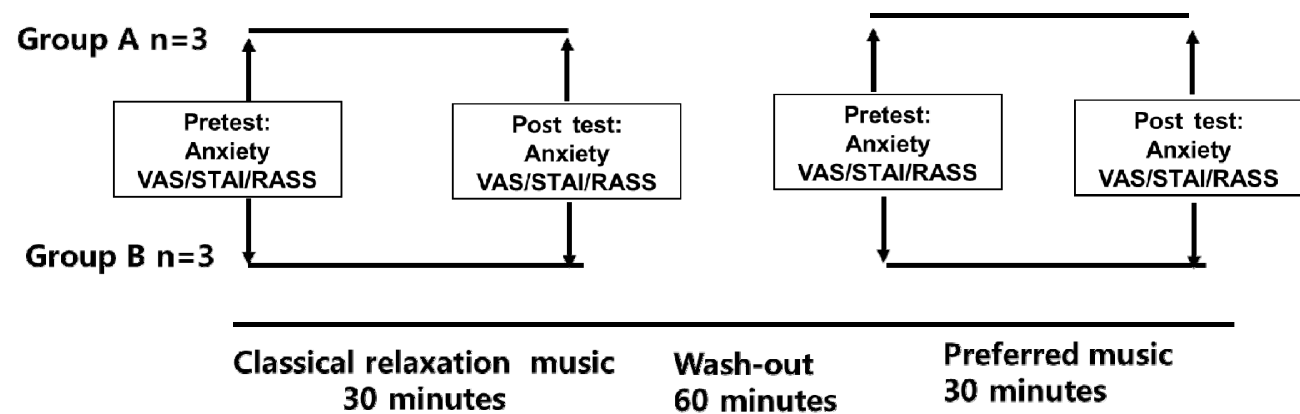

Preferred music 30 minutes

STAI=State-Trait Anxiety Inventory; VAS=Visual Analog Scale; RASS=Richmond Agitation Sedation Scale .

Figure 1. Flow chart of study design. 
Table 1. Patient Demographics

$(N=6)$

\begin{tabular}{llc}
\hline Variables & Categories & $\mathrm{n}(\%)$ or M \pm SD \\
\hline Gender & Male & $2(33.3)$ \\
& Female & $4(66.7)$ \\
Age & Range (20 60) & $45.33 \pm 16.49$ \\
Education & Bachelor's degree & $4(66.7)$ \\
& High school graduate & $2(33.3)$ \\
Job & Office worker & $3(50.0)$ \\
& Housewife & $1(16.7)$ \\
& None & $2(33.3)$ \\
Religion & Catholic & $1(16.7)$ \\
& Buddhist & $1(16.7)$ \\
& None & $4(66.6)$ \\
\hline
\end{tabular}

ted by any participant as his/her preferred music (Table 2).

\section{Pre/Post Comparison of Agitation and Anxiety}

Comparisons on anxiety and agitation between the preferred music intervention and classical relaxation music intervention are presented in Figure 2. Median RASS scores were significantly lower after both the preferred music intervention $(Z=-2.24, p=.025)$ and classical relaxation music intervention $(Z=-2, p=.046)$ compared to before. Patients showed moderate anxiety on the VAS and borderline anxiety on the STAI before the music interventions. Both the VAS and STAI scores decreased significantly after the preferred music intervention $(Z=-2.07, p=.038 ; Z=-2.21, p=$ .027). Differences in median VAS and STAI scores were also statistically significant after the classical relaxation music intervention compared to before music intervention $(Z=-2.27, p=.023 ; Z=-2.21, p=.024)$. There was no significant difference in the decrease in median anxiety scores (either VAS or STAI) between the two music interventions $(\mathrm{U}=15, p=.589 ; \mathrm{U}=9, p=.145)$ and in median RASS scores (U=15, $p=.523)$ (Table 3).

Furthermore, $83 \%$ of patients after the preferred music intervention and $67 \%$ after the classical relaxation music were alert and calm (i.e., had a score of zero on the RASS). Regarding the STAI, $50 \%$ and $67 \%$ of patients after the preferred and classical relaxation music interventions, respectively, showed decreased anxiety.

\section{Feasibility: Patients' Responses during the Music Interventions}

One of the patients demonstrated a change in agitated behaviors after music interventions. She tried to take off a medical device before listening to the music, but she stop-

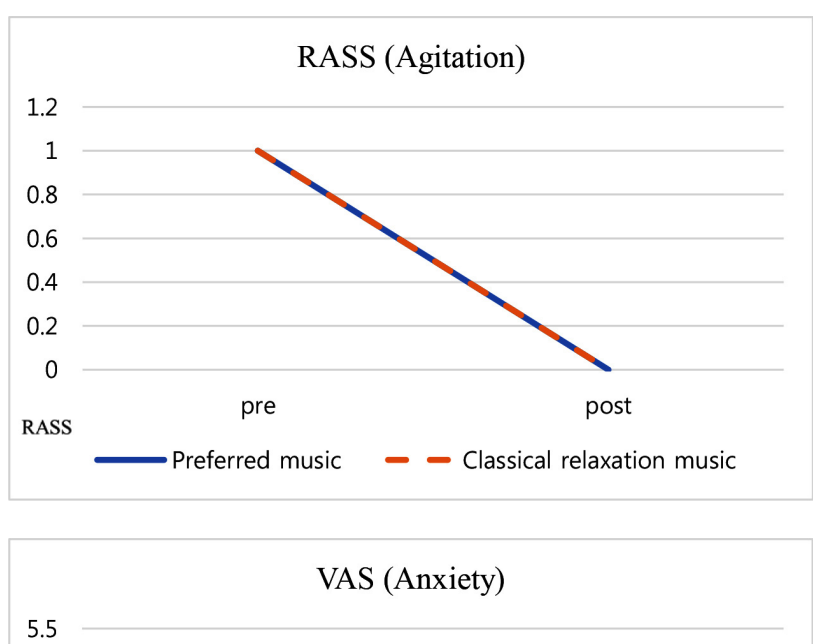

5.5
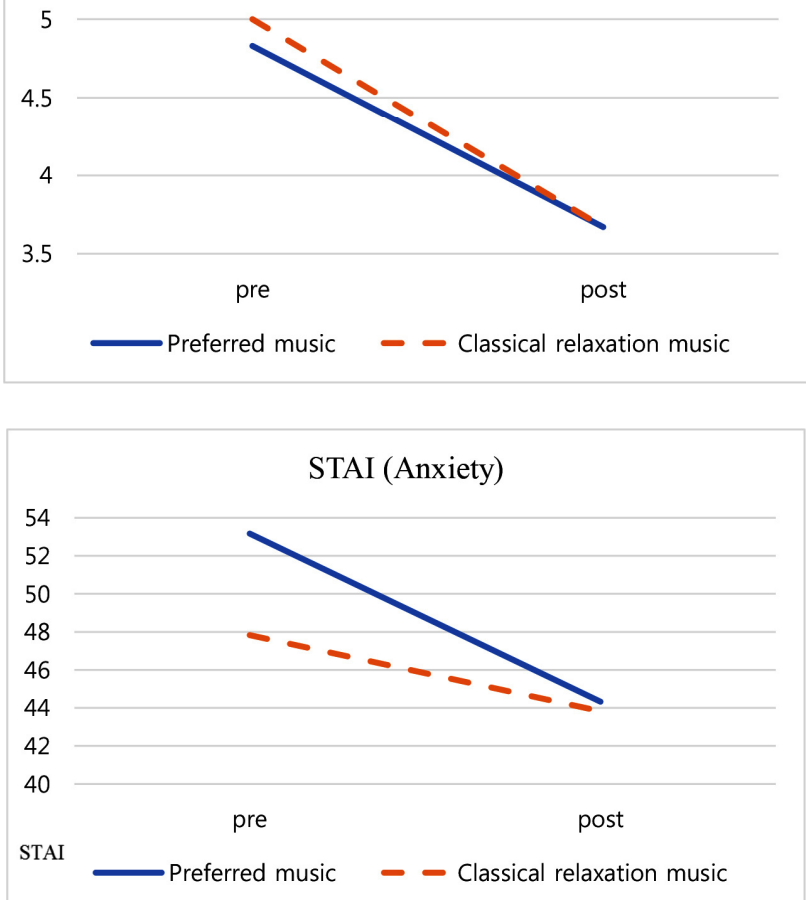

Figure 2. Pre-post comparisons of agitation and anxiety scores.

ped and quietly listened to the preferred music. She was very calm for the rest of the protocol. Another patient was smiling and moving his lips along with the lyrics while listening.

\section{DISCUSSION}

Both preferred music and classical relaxation music were used to relieve agitation and anxiety among patients during MV weaning. Results revealed that both types of 
Table 2. Clinical Characteristics and Preferred Music List

\begin{tabular}{|c|c|c|c|c|c|}
\hline Patient & Age & Gender & Medical diagnosis & Days of MV & Preferred music genre: song title \\
\hline 1 & 44 & Female & Hemoperitoneum & 4 & K-pop song: "Some" and "Travel" by BOL4 \\
\hline 2 & 32 & Female & Gestational hypertension & 2 & $\begin{array}{l}\text { K-pop songs: "Let it rain", "Drifting apart," and } \\
\text { "Loop" by NELL }\end{array}$ \\
\hline 3 & 60 & Male & Obstructive ileus & 17 & $\begin{array}{l}\text { Korean oldies music: "A woman out of a } \\
\text { window" and "Bounce" by Jo Yong-Pil }\end{array}$ \\
\hline 4 & 20 & Female & $\begin{array}{l}\text { Fracture of distal femur and } \\
\text { knee (left) }\end{array}$ & 35 & K-pop song: "Galaxy" by BOL4 \\
\hline 5 & 57 & Female & Advanced gastric cancer & 4 & $\begin{array}{l}\text { Korean oldies music: "Mona Lisa" and "The } \\
\text { dreams" by Jo Yong-Pil }\end{array}$ \\
\hline 6 & 59 & Male & $\begin{array}{l}\text { Coronary artery obstructive } \\
\text { disease }\end{array}$ & 2 & Pop music: "You raise me up" by Josh Groban \\
\hline
\end{tabular}

$\mathrm{MV}=$ mechanical ventilation.

Table 3. Medians of Agitation and Anxiety before and after Classical Relaxation Music and Preferred Music Intervention $(N=6)$

\begin{tabular}{|c|c|c|c|c|c|}
\hline \multirow{3}{*}{ Variables } & \multirow{3}{*}{ Categories } & \multicolumn{2}{|c|}{ Classical relaxation music } & \multicolumn{2}{|c|}{ Preferred music } \\
\hline & & Pretest & Posttest & Pretest & Posttest \\
\hline & & Median & Median & Median & Median \\
\hline Anxiety & $\begin{array}{l}\text { VAS } \\
\text { STAI }\end{array}$ & $\begin{array}{c}5 \\
47.5\end{array}$ & $\begin{array}{c}4 \\
45\end{array}$ & $\begin{array}{c}4.5 \\
54.5\end{array}$ & $\begin{array}{c}4 \\
46\end{array}$ \\
\hline Agitation & RASS & 1 & 0 & 1 & 0 \\
\hline
\end{tabular}

music were effective for improving agitation and anxiety. It is difficult to compare these findings with previous work, as no prior studies have assessed the effect of music therapy on agitation during the weaning process. However, similar results have been found in previous studies-for example, relaxing music therapy led to a reduction in agitation among intubated patients [27], and naturebased sound therapy decreased agitation during MV weaning [19]. Music stimulation might suppress agitated behavior by influencing the gamma aminobutyric acid (GABA) receptor pathway. GABA is one of the major inhibitory neurotransmitter receptors in the brain [28], and GABA can become synchronized to music stimuli, resulting in a calming effect [29]. Overall, our results could be used as a foundation for future research on the effect of music interventions on agitation.

Patients' anxiety also significantly decreased after both music interventions. This finding is consistent with previous studies showing that patients participating in patient-directed music interventions [18] and classical music interventions $[16,17]$ have lower anxiety levels. This could perhaps be due to preferred music being a positive envi- ronmental stimulus that can elicit pleasing memories and positive emotions through integration with individuals' personal lives [30]. Classical music, on the other hand, can facilitate a relaxation response, making classical music effective for reducing anxiety among patients weaning from MV [8].

The intervention effects for agitation and anxiety did not significantly differ between the preferred music and classical relaxation music interventions. Potentially, the lack of a significant difference was related to the small sample size. Therefore, it is recommended that the effect of preferred music on agitation and anxiety during the weaning process of MV is examined in a larger sample in the future.

Results regarding patients' responses during the music interventions suggest that music can have a calming effect on behavior, as well. Patients were much quieter and calmer before listening to the music, and some patients were quite immersed in the activity. This is consistent with a previous study demonstrating that patients listening to preferred music displayed a positive response by keeping time with the music and singing along [13]. 


\section{Study limitations and suggestions for future research}

Limitations should be noted. First, the study was conducted in one ICU in South Korea with a small patient sample. Therefore, the results cannot be generalized to all patients dealing with the MV weaning process. Future studies should attempt to replicate our findings in a sufficiently larger sample across various hospitals in South Korea.

Second, the music intervention was provided to patients only one time, which might have obscured any possible differences between the preferred music and classical relaxation music interventions. Participants also expressed that it was fun to listen to the preferred music, but they were disappointed that they could only listen to the preferred music once. Therefore, conducting the music interventions repeatedly over a longer duration, to determine whether patients will respond differently to these types of music, may be necessary.

Despite these limitations, the present findings provide practical evidence that both preferred music and classical relaxation music may be effective for decreasing agitation and anxiety during the weaning process. Up until now, there was no compelling evidence of an

advantage to music therapy in reducing agitation, and no prior study compared the effectiveness of different music types. Therefore, music that either considers a patient's preferences or has a calming effect should be provided for patients struggling with the MV weaning process. Furthermore, reducing patient anxiety and agitation is also beneficial for nurses who are trying to perform challenging procedures. To better apply similar interventions in a clinical setting, helping patients select preferred music and creating an ICU environment that is suitable for music listening is necessary. Given that general ICU, environments are quite noisy and stimulating, patients may find it difficult to concentrate on the music. Therefore, nurses should consider a patient's daily schedule as to what would be appropriate for listening to music and means for mitigating noise.

\section{CONCLUSION}

The present study revealed that music interventions resulted in a reduction in agitation and anxiety during the MV weaning process. All patients were extubated successfully; thus, music appears to be a safe, easy, and inexpensive intervention for healthcare providers to employ. Therefore, music might be useful as a non-pharmacological agent for MV patients. This feasibility study is meaningful in that it showed, for the first time, that music inter- ventions during the weaning process could lead to decreased agitation. Future studies are needed to identify whether there is, in fact, a difference between preferred music and other types of music, as well as extending these findings to larger and more diverse sample pools.

\section{REFERENCES}

1. Han CH, Kim YS, Park EC, Lee SH, Hong JH, Kim DW. Analysis of the factors related to the status of patients in the intensive care unit, medical utilization, survival rate, and prognosis using the data from the National Health Insurance claims. Gyeonggi-do: Institute of Health Insurance and Clinical Research in Ilsan Hospital, 2017 December. Report No.: 2017-20-026.

2. Girard TD, Kress JP, Fuchs BD, Thomason JW, Schweickert WD, Pun BT, et al. Efficacy and safety of a paired sedation and ventilator weaning protocol for mechanically ventilated patients in intensive care(Awakening and Breathing Controlled trial): a randomized controlled trial. The Lancet. 2008;371(9607): 126-134. https://doi.org/10.1016/S0140-6736(08)60105-1.

3. Rose L, Nonoyama M, Rezaie S, Fraser I. Psychological wellbeing, health-related quality of life and memories of intensive care and a specialized weaning center reported by survivors of prolonged mechanical ventilation. Intensive Critical Care Nursing. 2014;30(3):145-151.

https://doi.org/10.1016/j.iccn.2013.11.002.

4. De Wit M, Miller KB, Green DA, Ostman HE, Gennings C, Epstein SK. Ineffective triggering predicts increased duration of mechanical ventilation. Critical Care Medicine. 2009;37(10): 2740-2745. https://doi.org/10.1097/CCM.0b013e3181a98a05

5. Seymour CW, Martinez A, Christie JD, Fuchs ED. The outcome of extubation failure in a community hospital intensive care unit: a cohort study. Critical Care. 2004;8:R322-327. https://doi.org/10.1186/cc2913

6. Blackwood B, Alderdice F, Burns K, Cardwell C, Lavery G, $\mathrm{O}$ 'Halloran $\mathrm{P}$. Use of weaning protocols for reducing duration of mechanical ventilation in critically ill adult patients: Cochrane systematic review and meta-analysis. The British Medical Journal. 2011;342:c7237.

https://doi.org/10.1136/bmj.c7237

7. Tracy MF, Chlan L. Nonpharmacological interventions to manage common symptoms in patients receiving mechanical ventilation. Critical Care Nurse. 2011;31(3):19-28. https://doi.org/10.4037/ccn2011653.

8. Elbouhy MS, AbdelHalim HA, Hashem AMA. Effect of respiratory muscles training in weaning of mechanically ventilated COPD patients. Egyptian Journal of Chest Diseases and Tuberculosis. 2014;63(3):679-687.

https://doi.org/10.1016/j.ejcdt.2014.03.008. 
9. Gopal A, Roberts KJ, Anderson E, Siegel AM. Ventilator dependent patients successfully weaned with cognitive behavioral therapy: a case series. Psychosomatics; 2019, online. https://doi.org/10.1016/j.psym.2019.02.003

10. Hetland B, Lindquist R, Weinert CR, Peden-McAlpine C, Savik $K$, Chlan L. Predictive associations of music, anxiety, and sedative exposure on mechanical ventilation weaning trials. American Journal of Critical Care. 2017;26(3):210-220. https://doi.org/10.4037/ajcc2017468

11. Brush DR, Kress JP. Sedation and analgesia for the mechanically ventilated patient. Clinical in Chest Medicine. 2009;30 (1):131-41. https://doi.org/10.1164/rccm.201102-0273CI

12. Davis WB, Gfeller KE, Thaut MH. An introduction to music therapy: theory and practice. 3rd ed. Silver Spring MD: The American Music Therapy Association; 2008.

13. Park S, Williams RA, Lee D. Effect of preferred music on agitation after traumatic brain injury. Western Journal of Nursing Research. 2016;38(4):394-410. https://doi.org/10.1177/0193945915593180

14. Lee OK, Chung YF, Chan MF, Chan WM. Music and its effect on the physiological responses and anxiety levels of patients receiving mechanical ventilation: a pilot study. Journal of Clinical Nursing. 2005;14(5):609-620. https://doi.org/10.1111/j.1365-2702.2004.01103.x

15. Bradt J, Dileo C, Grocke D. Music interventions for mechanically ventilated patients. Cochrane Database Systematic Review. 2014;12:CD006902. https://doi.org/10.1002/14651858.CD006902.pub3.

16. Synn AR, Choe ME. Effect of music therapy on the physiological index, anxiety and dyspnea of patients with mechanical ventilator weaning. Journal of Korean Biological Nursing Science. 2012;14(1):57-65. https://doi.org/10.7586/jkbns.2012.14.1.57

17. Neha S. Effectiveness of music therapy on level of anxiety among patients weaned from mechanical ventilator in selected hospital at Meerut. International Journal of Science and Research. 2018;7(5):1477-1482.

18. Liang Z, Ren D, Choi J, Happ MB, Hravnak M, Hoffman LA. Music intervention during daily weaning trials- A 6 day prospective randomized crossover trial. Complementary Therapies in Medicine. 2016;29:72-77. https://doi.org/10.1016/j.ctim.2016.09.003

19. Saadatmand V, Rejeh N, Heravi-Karimooi M, Tadrisi SD, Zayeri F, Vaismoradi M, et al. Effect of nature-based sounds' intervention on agitation, anxiety, and stress in patients under mechanical ventilator support: a randomized controlled trial. International Journal of Nursing Studies. 2013;50(7):895-904. https://doi.org/10.1016/j.ijnurstu.2012.11.018

20. Piotto RF, Maia LN, Machado MN, Orrico SP. Effects of the use of mechanical ventilation weaning protocol in the Coronary Care Unit: randomized study. Brazilian Journal of Cardiovascular Surgery. 2011;26(2):213-221. https://doi.org/10.1590/S0102-76382011000200011

21. Chlan LL, Heiderscheit A. A tool for music preference assessment in critically ill patients receiving mechanical ventilatory support. Music Therapy Perspectives. 2009;27(1):42-47. https://doi.org/10.1093/mtp/27.1.42

22. Sessler CN, Gosnell MS, Grap MJ, Brophy GM, O'Neal PV, Keane KA, et al. The Richmond agitation sedation scale validity and reliability in adult intensive care unit patients. American Journal of Respiratory and Critical Care Medicine. 2002; 166(10):1338-1344. https://doi.org/10.1164/rccm.2107138

23. Spielberger CD, Gorsuch RL, Lushene R, Vagg PR, Jacobs GA. Manual for the State-Trait Anxiety Inventory. Palo Alto, CA: Mind Garden, Inc; 1983.

24. Hetland B, Lindquist R, Chlan LL. The influence of music during mechanical ventilation and weaning from mechanical ventilation: A review. Heart \& Lung. 2015;44(5):416-425. https://doi.org/10.1016/j.hrtlng.2015.06.010.

25. Quek KF, Low WY, Razack AH, Loh CS, Chua CB. Reliability and validity of the Spielberger State-Trait Anxiety Inventory (STAI) among urological patients: a Malaysian study. The Medical Journal of Malaysia. 2004;59(2):258-267.

26. Gerdner LA. Effects of individualized versus classical relaxation music on the frequency of agitation in elderly persons with Alzheimer's disease and related disorders. International Psychogeriatrics. 2000;12:49-65. https://doi.org/10.1017/S1041610200006190

27. Jaber S, Bahloul H, Guétin S, Chanques G, Sebbane M, Eledjam JJ. Effects of music therapy in the intensive care unit without sedation in weaning patients versus non-ventilated patients. Annales Françaises d'Anesthésie et de Réanimation. 2007;26 (1):30-38. https://doi.org/10.1016/j.annfar.2006.09.002

28. Kim KJ, Lee SN, Lee BH. Music therapy inhibits morphineseeking behavior via GABA receptor and attenuates anxietylike behavior induced by extinction from chronic morphine use. Neuroscience Letters. 2018;674(1):81-87. https://doi.org/10.1016/j.neulet.2018.03.035

29. Tylor DB. Biomedical functions of music as therapy. 2nd ed. St Louis, MO: Barton Publications; 2010.

30. Gerdner LA. An individualized music intervention for agitation. Journal of the American Psychiatric Nurses Association. 1997; 3:177-184. https://doi.org/10.1016/S1078-3903(97)90043-4 